\title{
Can theorems help treat cancer?
}

\author{
Jean Clairambault ${ }^{1}$ \\ INRIA Paris-Rocquencourt, BANG project-team, BP 105, F78153 Le Chesnay Cedex \\ and Laboratoire Jacques-Louis Lions, UPMC, 4 Place Jussieu, F75005 Paris
}

Can mathematicians who prove theorems be useful to physicians who treat patients with cancer? Can mathematics have an impact on cancer biology? Is mathematics more than just a tool in the hands of [a few open-minded] physicians willing to understand and treat cancer from an innovative point of view? ... and is mathematical biology more than just a toy in the hands of mathematicians? I imagine that most readers of this journal would join me in answering "yes" to these questions. But let me be more explicit in my questioning: Is there a place for a very abstract theoretical activity, as is proving theorems, in a team of physicians daily committed to treating human diseases (in particular cancer)? Can these physicians see the contribution of mathematicians to their difficult task as more than helping them to "think differently" (as some of them state it), but further as actually taking part in a therapeutic decision? As regards mathematical biology, when it is applied to medical questions, do we mathematicians feel enough committed to go beyond a few publications in the numerous journals dedicated to applications of maths to biology, and to collaborate with medical teams in a long term effort to improve in common medical treatments? Building on recent articles [3,4] in which I have outlined technical ways by which one can achieve a working collaboration between mathematicians and physicians toward such mutual usefulness, I will explain briefly below why the answers to these questions should be "yes".

At present, biologists and physicians do not look to mathematicians for theorems. An obvious reason for this is the completely different status of knowledge in mathematics and biology and medicine. Unlike mathematics, biology is a field where little is known for certain, for the very nature of the phenomena under study is often inaccessible, and deductive reasoning about uncertain facts of questionable value. Moreover, theorems as such do not exist in biology, although it is not impossible that theorems proven in a mathematical framework were inspired by problems posed initially as biological questions. The clinical relevance of such theoretical results is, however, seldom clear. More precisely, if our aim is to identify optimal schedules for the treatment of cancers by combining different drugs and taking account of cell cycle phase times, it is possible theoretically to determine optimal schedules for the delivery of drugs (with at least a theorem of existence for such an optimal schedule, and numerically determined schedules, as proposed for instance in [1]). In practice it is not easy to establish whether the assumptions that must hold for the theorems and optimal solutions to be valid are fulfilled - for they rely on the measurement of

\footnotetext{
${ }^{1}$ E-mail: jean.clairambault@inria.fr
} 
intracellular quantities that are difficult, or even impossible, to obtain. As a result, the practical application of mathematical results may depend upon biological 'facts' that, as is usual in biology, may be very likely, but are seldom certain.

This does not, however, mean that theorems are of no use to physicians. Firstly measurements that can not be made today may tomorrow become routine (consider, for example, the determination of cell cycle phases in individual proliferating cells using fluorescence markers [6], which until recently was unimaginable). Secondly, when presented with physiologically based sets of competing hypotheses, mathematicians can use theoretical models to compare scenarios and to identify those which best (in some sense) reproduce the biological system under investigation. For instance, in a therapeutic setting, theoretical exploration of alternative model scenarios may reveal that a given treatment strategy must be forsaken in favour of one which always yields better results.

The impact of mathematics in cancer medicine is at its best when it is based on genuine collaborations between mathematicians and clinicians and typically operates in one of two different ways. On the one hand, we have model-based predictive representations of tumour growth without treatment, or of its molecular counterparts at the intracellular level - this I will term the physicist's, or descriptive, point of view. On the other hand we have theoretical optimisation of cancer treatments - which I will call the physician's, or interventionistic, point of view. These alternative directions reflect the fact that cancer medicine does not deal only with the so-called 'natural history of cancers' (an expression borrowed from the vocabulary of medical schools, which means the description of cancer progression in the absence of medical intervention); the obvious main objective of medicine is to heal and, where possible, to cure patients with cancer.

This alternative is not a philosophical or moral consideration about a right or wrong way to act; it is plainly practical. It would certainly be delusional to hope to describe normal physiology before considering perturbations associated with diseased states, since medical progress has never proceeded in this way: physiology has always made its major advances by exploiting insight from pathology. It is indeed from neuropathological observations that both Broca and Wernicke, long before functional cerebral imagery could even be dreamt of, discovered the cerebral areas of language that now bear their names. In the same way, in my opinion, it would be equally delusional, mutatis mutandis, to hope to describe diseases in an efficient manner, from a medical viewpoint, i.e., with the aim to cure patients, without taking account of previous successes and failures of [mainly pharmacological] treatments - a view already expressed in [2] -, which may be considered as perturbations of 'natural' physiopathology. It is only in the limited field of biology considered independently of medicine (which some biologists even regard as a plain "applied branch" of their discipline, a rather extreme attitude) that the first strictly descriptive viewpoint may, to an equally limited extent, be justified in cancer modelling.

Defining a formalism that will help mathematicians better to understand the phenomena at stake in cancer (cautiously avoiding claiming to 'explain' these phenomena to biologists and physicians, an attitude that is seldom appreciated by them and will very likely spoil a working relationship; this is a personal viewpoint also presented in [5]) is thus more efficient when it is firstly thought of as 'what to do?', i.e., modelling in order to control what and by which means on which targets. Most oncologists are sensitive to this argument, at least those who consider mathematicians as possible contributors to therapeutic improvements. Among the others, the "physicist's 
viewpoint" may be more popular, limiting collaborative work to the descriptive biology of cancer, which may also, of course, have therapeutic consequences, predicted or not, and will certainly not be neglected by physicians. These arguments lead me to answer "yes" to the second question too: either directly through active collaboration with oncologists to optimise cancer treatments, or indirectly, by focussing on cancer biology, mathematicians can certainly have a positive impact on physicians dealing with cancer, making them think differently and possibly opening new therapeutic tracks.

In the same way, I will answer the third question positively: mathematics is not a tool in the hands of physicians, for they deal with mathematicians, rather than mathematics. With mutual interest on both sides, both increased understanding and practical improvements can emerge from such collaborations. As regards the attitude of mathematicians themselves to mathematical biology (fourth question), I would say that it depends primarily upon the attitude of the mathematicians: either they use mathematical biology as a source of mathematical problems and care little about applications, or they actively seek out like-minded physicians in order to address problems of genuine biomedical importance, to cast them in a mathematical framework and to ensure that the impact of theoretical solutions is fully exploited for practical, therapeutic purposes. The latter attitude is the most demanding in terms of commitment and patience, but the one that will bear the richest fruits for both parties. Needless to say also, a third way that involves conjugating where possible, the search for nice mathematical problems with a commitment to therapeutic enterprises, without opposing them, is clearly the most satisfying for the mathematician and for the physician alike.

\section{References}

[1] Basdevant, C., Clairambault, J., Lévi, F. Optimisation of time-scheduled regimen for anticancer drug infusion. Mathematical Modelling and Numerical Analysis, 39(6):1069-1086, 2005 .

[2] Bernard, Claude. Introduction à l'étude de la médecine expérimentale, p. 1. J. B. Baillière et fils, Paris, 1865.

[3] Clairambault, J. Modelling physiological and pharmacological control on cell proliferation to optimise cancer treatments. Mathematical Modelling of Natural Phenomena, 4(3):12-67, 2009.

[4] Clairambault, J. Optimising cancer pharmacotherapeutics using mathematical modelling and a systems biology approach. Personalized Medicine, 8(3):271-286, 2011.

[5] Clairambault, J. Commitment of mathematicians in medicine. A personal experience, and generalisations. Acta Biotheoretica, 59(3):201-211, 2011.

[6] Sakaue-Sawano, A., Ohtawa, K., Hama, H., Kawano, M., Ogawa, M., Miyawaki, A. Tracing the silhouette of individual cells in S/G2/M phases with fluorescence. Chemistry \& Biology, 15:1243-48, 2008. 